Research Article

\title{
Exponential Stability of Impulsive Stochastic Delay System Based on Razumikhin Method and Its Application to Chaos Control
}

\author{
Shiguo Huang $\left(\mathbb{0},{ }^{1}\right.$ Yujun Niu, ${ }^{2}$ and Yajing $\mathrm{Xu}^{1}$ \\ ${ }^{1}$ Department of Mathematics and Information Science, Zhengzhou University of Light Industry, Zhengzhou 450002, China \\ ${ }^{2}$ School of Mathematics and Science, Nanyang Institute of Technology, Nanyang 473004, China \\ Correspondence should be addressed to Shiguo Huang; financialmath@126.com
}

Received 22 June 2021; Accepted 11 August 2021; Published 23 August 2021

Academic Editor: Eric Campos

Copyright (c) 2021 Shiguo Huang et al. This is an open access article distributed under the Creative Commons Attribution License, which permits unrestricted use, distribution, and reproduction in any medium, provided the original work is properly cited.

In this paper, the exponential stability of a stochastic delay system with impulsive signal is considered, and stability theorem of this system is proposed based on the Lyapunov-Razumikhin method; the convergence rate is also given, which gives theoretical foundation to chaos control and synchronization using the impulsive method. Finally, the classic delay chaos system with white noise and impulsive signal is employed to verify the feasibility and effectiveness of our theorem.

\section{Introduction}

The control of dynamic systems, including but not limited to nonstationary control [1], chaos control [2], etc., has always been an important topic. The impulsive method is an important control scheme, especially for chaos control. It can obtain stability and synchronization using only small signal, which is very important when the system cannot tolerate continuous disturbance or the control signal is expensive. Many scholars studied impulsive phenomenon [3-13]. Yang [7] and Sun and Zhang [9] made constructive contribution in the determinate impulsive system. $\mathrm{Wu}$ [14] studied the $P$-moment stability of a jumping system. Cao et al. $[15,16]$ investigated the Pth moment exponential stability for stochastic functional differential equations under different conditions. Li et al., Niu et al., and some other scholars [17-21] investigated the stability of impulsive stochastic differential equations and its application to chaos control and synchronization using the impulsive method. In addition, delay is a common phenomenon in nonlinear science, which can induce complex dynamic action and has aroused the strong interest of many scholars. Yang et al. [22, 23] studied the mean stability and exponential $P$-stability of impulsive stochastic differential equations with delays. We know that the Lyapunov-Razumikhin method is an important and effective tool to study the stability of delay differential equations. Yan and Shen [24] and Wang and Liu [25] investigated the impulsive stabilization of a class of delay differential equations, and numerical examples were given to verify the method mentioned in their work. Unfortunately, for the impulsive stochastic delay differential system, there is no theory about the stability using the Razumikhin method, which gives theoretical guarantee to impulsive control and synchronization in the stochastic delay system. In this paper, a judgement theorem about impulsive stochastic delay differential equations based on the Lyapunov-Razumikhin method is deduced, and chaos control of stochastic Lorenz system using the impulsive method is employed to verify the effectiveness of our theorem.

\section{Preliminary Notes and Definitions}

Let $R^{n}$ be an $n$-dimensional Euclidean space with the norm $\|\cdot\| . R^{n \times m}$ is the set of all $n \times m$ matrices, $R_{+}=\{x: x \in$ $R, x \geq 0\}, R_{t_{0}}=\left\{t: t \in R_{+}, t \geq t_{0}\right\}$, and $S_{\rho_{0}}=\left\{X \in R^{n} \mid\|X\|<\right.$ $\left.\rho_{0}\right\}$. The family of all nonnegative functions from $R_{t_{0}} \times R^{n}$ to $R_{+}$is denoted by $C^{1,2}\left(R_{t_{0}} \times R^{n}, R_{+}\right)$, of which these functions 
are first-order continuously differentiable with respect to the first variable and second-order differentiable with respect to the second variable. $\|\psi\|_{\tau}=\sup _{-\tau \leq s \leq 0} E\|\psi(s)\|$, for $\psi \in P C\left([-\tau, 0], R^{n}\right), \tau>0$, where $E(X)$ means the expectation of $X$ and $P C\left([-\tau, 0], R^{n}\right)$ denotes the family of all piecewise right continuous functions.

Consider the following impulsive stochastic delay system:

$$
\begin{cases}\mathrm{d} X(t)=F\left(t, X_{t}\right) \mathrm{d} t+G\left(t, X_{t}\right) \mathrm{d} W(t), & t \neq t_{k}, \\ \Delta X(t)=I_{k}\left(t_{k}, X_{t_{k}^{-}}\right), & k \in N, \\ X_{t_{0}}=X_{0}, & \end{cases}
$$

where $F, G, I_{k}: R_{t_{0}} \times P C\left([-\tau, 0], R^{n}\right) \longrightarrow R^{n}, W(t)$ is a normal $m$-dimensional Wiener process, $X_{0} \in P C\left(\left[\begin{array}{ll}-\tau, 0 & 0\end{array} R^{n}\right)\right.$, $0 \leq t_{0}<t_{1}<t_{2}<\cdots<t_{k}<\cdots, \lim _{k \longrightarrow \infty} t_{k}=\infty, t_{k}$ is the moment when impulse happens, $\Delta X(t)=X(t)-X\left(t^{-}\right), X_{i} \in P C([-\tau$, $\left.0], R^{n}\right)$, and $X_{i}(s)=X(i+s), i=t, t^{-}, s \in[-\tau, 0]$. We suppose there is a solution $X(t)=X\left(t, t_{0}, X_{0}\right)$ satisfying (1), which is left continuous: $X\left(\tau_{i}^{-}\right)=\lim _{t \longrightarrow \tau_{i}-0} X(t)=X\left(\tau_{i}\right)$ and has right limit: $X\left(\tau_{i}^{+}\right)=\lim _{t \rightarrow \tau_{i}+0} X(t)$.

Definition 1. Function $V: R_{t_{0}} \times R^{n} \longrightarrow R_{+}$belongs to class $\mathscr{V}_{0}$ if

(1) $V$ is continuous in each of sets $\left(\tau_{k-1}, \tau_{k}\right] \times R^{n}$, for all $t \geq 0, V(t, 0)=0$, and

$$
\lim _{(t, y) \longrightarrow\left(\tau_{k}^{+}, x\right)} V(t, y)=V\left(\tau_{k}^{+}, x\right) .
$$

(2) $V$ is locally Lipschitzian in $x \in R^{n}$.

Definition 2. For each $V(t, X) \in C^{1,2}\left(R_{t_{0}} \times R^{n}, R_{+}\right)$, define an operator $L$ from $R_{t_{0}} \times R^{n}$ to $R$ as $L V(t, X)=V_{t}(t, X)+V_{X}(t$, $X) f(t, X)+(1 / 2) \operatorname{trace}\left(g^{T}(t, X) V_{X X} g(t, X)\right)$, where $V_{t}(t$, $X)=(\partial V(t, X) / \partial t), \quad V_{X X}(t, X)=\left[\partial^{2} V(t, X) /\left(\partial x_{i} \partial x_{j}\right)\right]_{n \times n}$, and

$$
V_{X}(t, X)=\left[\frac{\partial V(t, X)}{\partial x_{1}}, \frac{\partial V(t, X)}{\partial x_{2}}, \ldots, \frac{\partial V(t, X)}{\partial x_{n}}\right] .
$$

Definition 3. Trivial solution of (1) is global exponentially stable if there is $\alpha>0, M \geq 1$ such that

$$
E\left(\left\|X\left(t, t_{0}, X_{0}\right)\right\|\right) \leq M\left\|X_{0}\right\|_{\tau} e^{-\alpha\left(t-t_{0}\right)}
$$

holds for $X_{t_{0}}=X_{0}$ and $\left(t, X_{0}\right) \in P C\left([-\tau, 0], R^{n}\right)$.

\section{Exponential Stability for Impulsive Stochastic Delay Differential Equations Based on Lyapunov-Razumikhin Method}

In this section, the Lyapunov-Razumikhin method is employed to study the exponential stability of impulsive stochastic delay differential equations, and judgement theorem of this problem will be deduced too.
Theorem 1. If there exists function $V \in \mathscr{V}_{0}$ and $p, c, c_{1}, c_{2}>0, \alpha>\tau, \lambda>c$ such that

(I) $c_{1} E\left(\|X\|^{p}\right) \leq E(V(t, X)) \leq c_{2} E\left(\|X\|^{p}\right)$ for $t \in R_{+}$ and $X \in R^{n}$;

(II) $E(L V(t, X)) \leq c E(V(t, X))$ holds for all $t \in\left[t_{k-1}\right.$, $\left.t_{k}\right), k \in N, \quad q \geq e^{2 \lambda \alpha}, \quad$ whenever $q E(V(t, X)) \geq E$ $\left(V\left(t+s, X_{t}(s)\right)\right)$ for $s \in[-\tau, 0]$;

(III) $E\left(V\left(t_{k}^{+}, X_{t_{k}}+I_{k}\left(t_{k}, X_{t_{k}}\right)\right)\right) \leq d_{k} E\left(V\left(t_{k}, X_{t_{k}}\right)\right)$, where $d_{k}>0, k \in N$;

(IV) $\tau \leq t_{k}-t_{k-1} \leq \alpha, \ln \left(d_{k}\right)+\lambda \alpha<-\lambda\left(t_{k+1}-t_{k}\right)$, then the trivial solution of system (1) is exponentially stable, and the convergence rate is $(\lambda / p)$.

Proof. Choose $M \geq 1$ such that

$$
\begin{aligned}
& c_{2} E\left(\left\|X_{0}\right\|_{\tau}^{p}\right)<M E\left(\left\|X_{0}\right\|_{\tau}^{p}\right) e^{-\lambda\left(t_{1}-t_{0}\right)-\alpha c} \\
& <M E\left(\left\|X_{0}\right\|_{\tau}^{p}\right) e^{-\lambda\left(t_{1}-t_{0}\right)} \leq q c_{2} E\left(\left\|X_{0}\right\|_{\tau}^{p}\right) .
\end{aligned}
$$

Let $X(t)=X\left(t, t_{0}, X_{0}\right)$ be the solution of system (1), $X_{t_{0}}=X_{0}$, and $V(t)=V(t, X)$. Then, we will prove that the following inequality holds:

$$
E(V(t)) \leq M E\left(\left\|X_{0}\right\|_{\tau}^{p}\right) e^{-\lambda\left(t_{k}-t_{0}\right)}, \quad t \in\left[t_{k-1}, t_{k}\right), k \in N .
$$

First, we will show that (6) holds for $k=1$, i.e.,

$$
E(V(t)) \leq M E\left(\left\|X_{0}\right\|_{\tau}^{p}\right) e^{-\lambda\left(t_{1}-t_{0}\right)}, \quad t \in\left[t_{0}, t_{1}\right) .
$$

From condition (I) and equation (5), for $t \in\left[t_{0}-\tau, t_{0}\right]$, we have

$$
\begin{aligned}
E(V(t)) & \leq c_{2} E\left(\|X\|^{p}\right) \leq c_{2} E\left(\|X\|_{\tau}^{p}\right)<M E\left(\|X\|_{\tau}^{p}\right) e^{-\lambda\left(t_{1}-t_{0}\right)-\alpha c} \\
& <M E\left(\|X\|_{\tau}^{p}\right) e^{-\lambda\left(t_{1}-t_{0}\right)} .
\end{aligned}
$$

If (7) is not true, there must be some $\bar{t} \in\left(t_{0}, t_{1}\right)$ such that

$$
\begin{gathered}
E(V(\bar{t}))>M E\left(\left\|X_{0}\right\|_{\tau}^{p}\right) e^{-\lambda\left(t_{1}-t_{0}\right)}>M E\left(\left\|X_{0}\right\|_{\tau}^{p}\right) e^{-\lambda\left(t_{1}-t_{0}\right)-\alpha c} \\
>c_{2} E\left(\left\|X_{0}\right\|_{\tau}^{p}\right) \geq V\left(t_{0}+s\right), \quad s \in[-\tau, 0] .
\end{gathered}
$$

As $V_{-}(t)$ is continuous in $\left(t_{0}, \bar{t}\right)$, there must exist $t^{*} \in\left(t_{0}, t\right)$ such that

$$
E\left(V\left(t^{*}\right)\right)=M E\left(\left\|X_{0}\right\|_{\tau}^{p}\right) e^{-\lambda\left(t_{1}-t_{0}\right)},
$$

and when $t \in\left[t_{0}-\tau, t^{*}\right]$,

$$
E(V(t)) \leq M E\left(\left\|X_{0}\right\|_{\tau}^{p}\right) e^{-\lambda\left(t_{1}-t_{0}\right)} .
$$

There exists $t^{* *} \in\left(t_{0}, \bar{t}\right)$ such that

$$
E\left(V\left(t^{* *}\right)\right)=c_{2} E\left(\left\|X_{0}\right\|_{\tau}^{p}\right) .
$$

When $t \in\left[t^{* *}, t^{*}\right]$, we have 


$$
E(V(t)) \geq c_{2} E\left(\left\|X_{0}\right\|_{\tau}^{p}\right) .
$$

So, for all $t \in\left[t^{* *}, t^{*}\right]$,

$$
\begin{aligned}
E(V(t+s)) & \leq M E\left(\left\|X_{0}\right\|_{\tau}^{p}\right) e^{-\lambda\left(t_{1}-t_{0}\right)} \leq q c_{2} E\left(\left\|X_{0}\right\|_{\tau}^{p}\right) \\
& \leq q E(V(t)), \quad s \in[-\tau, 0] .
\end{aligned}
$$

From condition (II), $L V(t) \leq c V(t)$ holds for $t \in\left[t^{* *}, t^{*}\right]$; then, we have

$$
\begin{gathered}
E\left(V\left(t^{* *}\right)\right) \geq E\left(V\left(t^{*}\right)\right) e^{-\alpha c}, \\
\text { i.e. } c_{2} E\left(\left\|X_{0}\right\|_{\tau}^{p}\right) \geq M E\left(\left\|X_{0}\right\|_{\tau}^{p}\right) e^{-\lambda\left(t_{1}-t_{0}\right)-\alpha c},
\end{gathered}
$$

which is in conflict with (5). In other words, (6) holds for $k=1$.

Suppose that (6) holds for $k=m, m \in N$, i.e.,

$$
E(V(t)) \leq M E\left(\left\|X_{0}\right\|_{\tau}^{p}\right) e^{-\lambda\left(t_{k}-t_{0}\right)}, \quad t \in\left[t_{k-1}, t_{k}\right),
$$

holds for $k=1,2, \ldots, m$. From condition (III) and (16), we know that

$$
\begin{aligned}
E\left(V\left(t_{m}\right)\right) & \leq d_{m} E\left(V\left(t_{m}^{-}\right)\right)<e^{-\lambda \alpha} e^{-\lambda\left(t_{m+1}-t_{m}\right)} M E\left(\left\|X_{0}\right\|_{\tau}^{p}\right) e^{-\lambda\left(t_{m}-t_{0}\right)} \\
& <M E\left(\left\|X_{0}\right\|_{\tau}^{p}\right) e^{-\lambda\left(t_{m+1}-t_{0}\right)} .
\end{aligned}
$$

In the following, we will prove that (16) holds for $k=m+1$, i.e.,

$$
E(V(t)) \leq M E\left(\left\|X_{0}\right\|_{\tau}^{p}\right) e^{-\lambda\left(t_{m+1}-t_{0}\right)}, \quad t \in\left[t_{m}, t_{m+1}\right) .
$$

If (18) is not true, let

$$
\bar{t}=\inf \left\{t \in\left[t_{m}, t_{m+1}\right) \mid E(V(t))>M E\left(\left\|X_{0}\right\|_{\tau}^{p}\right) e^{-\lambda\left(t_{m+1}-t_{0}\right)}\right\} .
$$

From (17), we know that $\bar{t} \neq t_{m}$. As $V(t)$ is continuous in $\left[t_{m}, t_{m+1}\right)$, we have

$$
E(V(\bar{t}))=M E\left(\left\|X_{0}\right\|_{\tau}^{p}\right) e^{-\lambda\left(t_{m+1}-t_{0}\right)},
$$

and when $t \in\left[t_{m}, \bar{t}\right]$,

$$
\begin{gathered}
E(V(t)) \leq M E\left(\left\|X_{0}\right\|_{\tau}^{p}\right) e^{-\lambda\left(t_{m+1}-t_{0}\right)}, \\
E\left(V\left(t_{m}\right)\right)<M E\left(\left\|X_{0}\right\|_{\tau}^{p}\right) e^{-\lambda\left(t_{m+1}-t_{0}\right)-\lambda \alpha}<E(V(\bar{t}))
\end{gathered}
$$

hold from (17). Then, there exists $t^{*} \in\left(t_{m}, \bar{t}\right)$, such that

$$
E\left(V\left(t^{*}\right)\right)=M E\left(\left\|X_{0}\right\|_{\tau}^{p}\right) e^{-\lambda\left(t_{m+1}-t_{0}\right)-\lambda \alpha} .
$$

Furthermore,

$$
E\left(V\left(t^{*}\right)\right) \leq E(V(t)) \leq E(V(\bar{t}))
$$

holds for $t \in\left[t^{*}, \bar{t}\right]$. As $\tau \leq t_{k}-t_{k-1} \leq \alpha, t+s \in\left[t_{m-1}, \bar{t}\right]$ holds for $t \in\left[t^{*}, t\right]$ and $s \in[-\tau, 0]$. We have the following inequality for $t \in\left[t^{*}, t\right]$ from (16) and (21):

$$
\begin{gathered}
E(V(t+s)) \leq M E\left(\left\|X_{0}\right\|_{\tau}^{p}\right) e^{-\lambda\left(t_{m}-t_{0}\right)}=M E\left(\left\|X_{0}\right\|_{\tau}^{p}\right) e^{-\lambda\left(t_{m+1}-t_{0}\right)} e^{\lambda\left(t_{m+1}-t_{m}\right)} \\
\quad \leq M E\left(\left\|X_{0}\right\|_{\tau}^{p}\right) e^{\lambda \alpha-\lambda\left(t_{m+1}-t_{0}\right)}=e^{2 \lambda \alpha} E\left(V\left(t^{*}\right)\right) \leq q E(V(t)), \quad s \in[-\tau, 0] .
\end{gathered}
$$

But from condition (II), (24), and $\lambda>c$, we know that

$$
\begin{aligned}
E(V(\bar{t})) \leq & E\left(V\left(t^{*}\right)\right) e^{\alpha c} \\
= & e^{-\lambda\left(t_{m+1}-t_{0}\right)-\lambda \alpha} M E\left(\left\|X_{0}\right\|_{\tau}^{p}\right) e^{\alpha c} \\
& <E(V(\bar{t})),
\end{aligned}
$$

which leads to a conflict, so (16) holds for $k=m+1$.

Therefore, (16) holds for all $k \in N$. From condition (I),

$$
E(\|X\|) \leq M^{*} E\left(\left\|X_{0}\right\|_{\tau}\right) e^{-(\lambda / p)\left(t-t_{0}\right)}
$$

holds for $t \in\left[t_{k-1}, t_{k}\right), k \in N$, where $M^{*}=\max \{1,(M /$ $\left.\left.c_{1}\right)^{(1 / p)}\right\}$. In other words, the trivial solution of system (1) is exponentially stable, and the convergence rate is $(\lambda / p)$.

\section{Numerical Example}

In order to show the validity and effectiveness of Theorem 1, we investigate chaos controllability of the Lorenz system with white noise and delay using the impulsive method in the sense of exponential stability.
The Lorenz system with delay and white-noise perturbation can be written as

$$
\left\{\begin{array}{l}
\dot{x}=\sigma(y-x)+\lambda_{1} x\left(t-t_{1}\right)+\beta x \frac{\mathrm{d} w(t)}{\mathrm{d} t}, \\
\dot{y}=r x-y-x z+\lambda_{2} x\left(t-t_{2}\right)+\beta y \frac{\mathrm{d} w(t)}{\mathrm{d} t}, \\
\dot{z}=-b z+x y+\lambda_{3} z\left(t-t_{3}\right)+\beta z \frac{\mathrm{d} w(t)}{\mathrm{d} t},
\end{array}\right.
$$

where $\sigma, r, b, \beta, \lambda_{1}, \lambda_{2}$, and $\lambda_{3}$ are all nonnegative parameters, $t_{1}, t_{2}$, and $t_{3}$ are nonnegative time delay parameters, and $w(t)$ is the normal Wiener process. When $\sigma=10, r=28$, $b=(8 / 3), \beta=0.5, \lambda_{1}=\lambda_{2}=\lambda_{3}=0.1$, and $t_{1}=t_{2}=t_{3}=$ $0.05,(28)$ is chaotic, and time history is given in Figure 1.

In the impulsive control scheme, signals are added to system (28) at discrete time $\tau_{i}, i=1,2, \ldots$, and system (28) suffers sudden changes at these instants, and this impulsive control scheme can be written as 


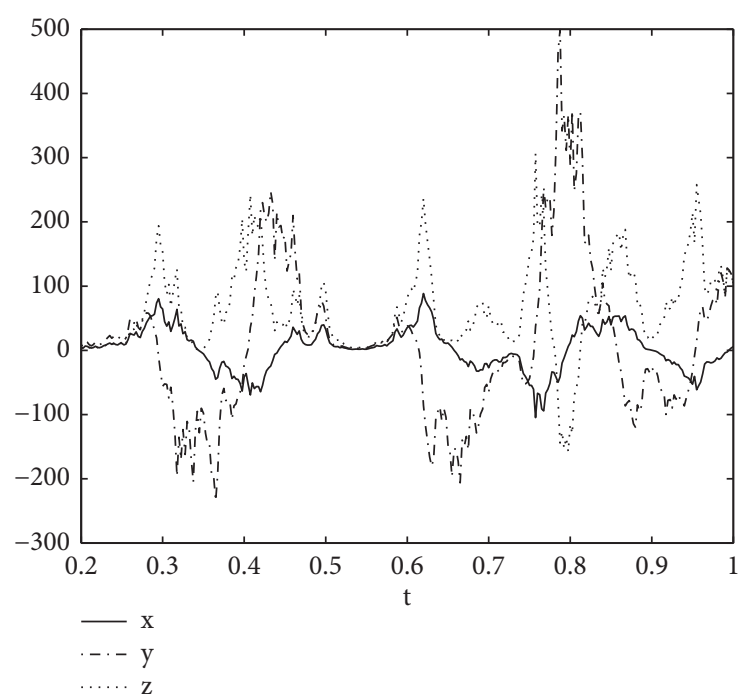

Figure 1: Time history of (28) under chaotic state.

$$
\left\{\begin{array}{l}
\dot{x}=-\sigma x+\sigma y+\lambda_{1} x\left(t-t_{1}\right)+\beta x \frac{\mathrm{d} w_{t}}{\mathrm{~d} t}, \\
\dot{y}=r x-y-x z+\lambda_{2} x\left(t-t_{2}\right)+\beta y \frac{\mathrm{d} w_{t}}{\mathrm{~d} t}, \\
\dot{z}=-b z+x y+\lambda_{3} x\left(t-t_{3}\right)+\beta z \frac{\mathrm{d} w_{t}}{\mathrm{~d} t}, \\
x\left(\tau_{i}^{+}\right)=\left(1+k_{1}\right) x\left(\tau_{i}\right), \\
y\left(\tau_{i}^{+}\right)=\left(1+k_{2}\right) y\left(\tau_{i}\right), \\
z\left(\tau_{i}^{+}\right)=\left(1+k_{3}\right) z\left(\tau_{i}\right),
\end{array}\right.
$$

where $k_{1}, k_{2}$, and $k_{3}$ are control parameters and $t \neq \tau_{i}$, $i=1,2, \ldots$. Suppose that impulses are equidistant, and $\tau_{i+1}-\tau_{i}=\delta, i=0,1,2, \ldots, \delta>0$. In the following, we will find out whether system (29) satisfies the conditions of Theorem 1, from which we can judge whether system (28) can be controlled using the impulsive method. Let $V(t, X)=x^{2}+y^{2}+z^{2}$, and it is easy to find that condition (I) of Theorem 1 holds.

$$
\begin{aligned}
E(L V(t, X))= & \left(\beta^{2}-2 \sigma\right) x^{2}+\left(\beta^{2}-2\right) y^{2}+\left(\beta^{2}-2 b\right) z^{2}+2(\sigma+r) x y \\
& +2 \lambda_{1} x\left(t-t_{1}\right) x+2 \lambda_{2} y\left(t-t_{2}\right) y+2 \lambda_{3} z\left(t-t_{3}\right) z \\
\leq & \left(\beta^{2}-\sigma+r+\lambda_{1}\right) x^{2}+\left(\beta^{2}-2+\sigma+r+\lambda_{2}\right) y^{2}+\left(\beta^{2}-2 b+\lambda_{3}\right) z^{2} \\
& +\lambda_{1} x^{2}\left(t-t_{1}\right)+\lambda_{2} y^{2}\left(t-t_{2}\right)+\lambda_{3} z^{2}\left(t-t_{3}\right) .
\end{aligned}
$$

Let $K=\max \left\{\left|\beta^{2}-\sigma+r+\lambda_{1}\right|, \quad\left|\beta^{2}+\sigma+r-2+\lambda_{2}\right|\right.$, $\left.\left|\beta^{2}-2 b+\lambda_{3}\right|\right\}$

$$
\lambda^{*}=\max \left\{\left|\lambda_{1}\right|,\left|\lambda_{2}\right|,\left|\lambda_{3}\right|\right\}
$$

and we have

$$
E(L(V(t, e))) \leq\left(\lambda^{*}+K\right) E(V(t, e)),
$$

so condition (II) of Theorem 1 holds. When $t=t_{k}, k=$ $1,2, \ldots, E\left(V\left(t_{k}, e_{t_{k}}+I_{k}\left(t_{k}+e_{t_{k}}\right)\right)\right)=\left(1+k_{1}\right)^{2} E\left(e_{x}^{2}\left(t_{k}\right)\right)+$ $\left(1+k_{2}\right)^{2} E\left(e_{y}^{2}\left(t_{k}\right)\right)+\left(1+k_{3}\right)^{2} E\left(e_{z}^{2}\left(t_{k}\right)\right)$, and let $d_{k}=\max$ $\left\{\left(1+k_{1}\right)^{2},\left(1+k_{2}\right)^{2},\left(1+k_{3}\right)^{2}\right\}$; then,

$$
E\left(V\left(t_{k}, e_{t_{k}}+I_{k}\left(t_{k}+e_{t_{k}}\right)\right)\right) \leq d_{k} E\left(V\left(t_{k}^{-}, e_{t_{k}}\right)\right)
$$

so condition (III) of Theorem 1 holds. In this example, let $k_{1}=-1.6, k_{2}=k_{3}=-1.1$, and we have $d_{k}=0.36$. Let $\lambda=4$, and thus, $\ln \left(d_{k}\right) \leq-2 \lambda \delta$, and then condition (IV) of Theorem 1 holds. According to Theorem 1, system (29) is exponentially stable, and the convergence rate is 2 . Figure 2 shows the evolution of $x(t), y(t)$, and $z(t)$, from which we 


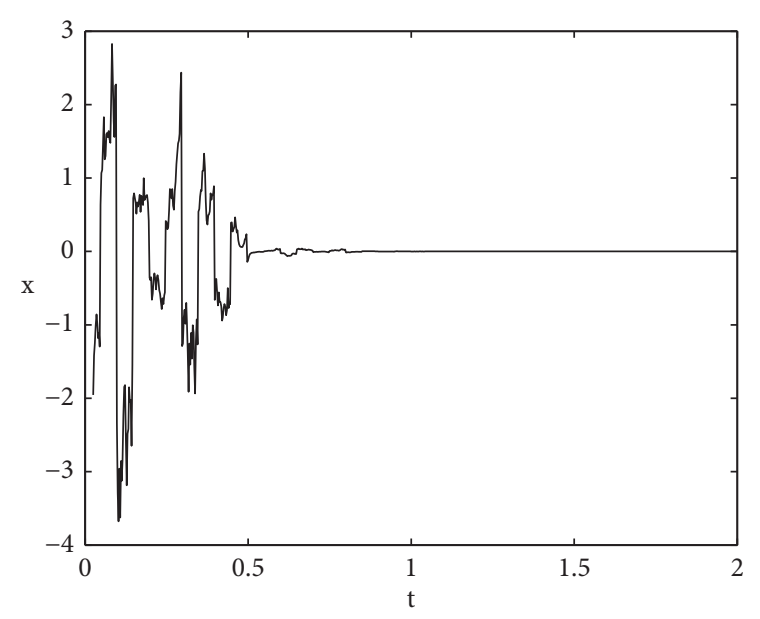

(a)

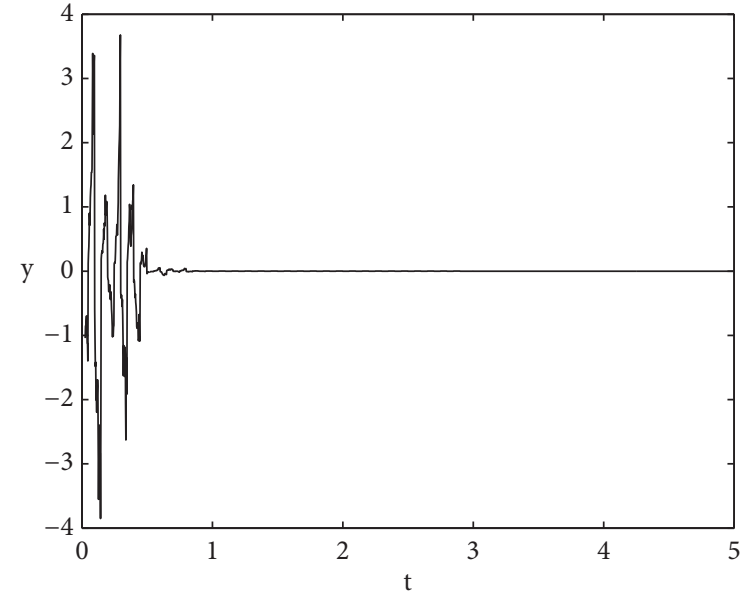

(b)

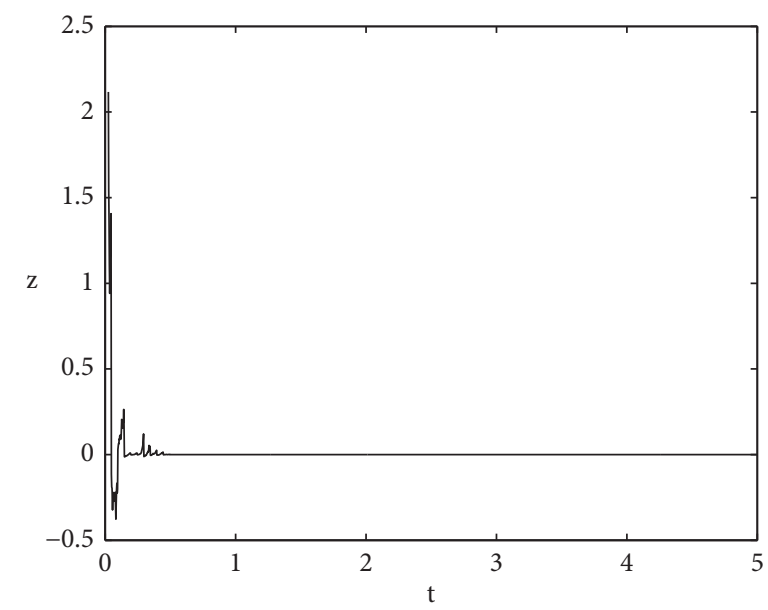

(c)

Figure 2: Time history of system (29) under stable state.

can find that system (29) approaches the origin as time goes by. In other words, system (28) can be controlled using the impulsive method.

\section{Conclusion}

In this paper, exponential stability of impulsive stochastic delay differential equations is studied using the Lyapunov-Razumikhin method, and a judgement theorem of Razumikhin form is given, which gives the theoretical foundation to chaos control and synchronization for the stochastic delay system using the impulsive method. The convergence rate of the control system can be deduced too. Impulsive control for the Lorenz system with white noise and time delay is taken as an example to show the validity and effectiveness of our theorem, and numerical simulation is employed to show the feasibility of the theoretical result.

\section{Data Availability}

The data used to support the findings of this study are available from the corresponding author upon request.

\section{Conflicts of Interest}

The authors declare that they have no conflicts of interest.

\section{Acknowledgments}

This study was supported by the National Natural Science Foundation of China (grant no. U1504105).

\section{References}

[1] J. Cheng, Y. Shan, J. Cao, and J. H. Park, "Nonstationary control for T-S fuzzy Markovian switching systems with variable quantization density," IEEE Transactions on Fuzzy Systems, vol. 29, no. 6, pp. 1375-1385, 2021.

[2] M. A. Akinlar, F. Tchier, and M. Inc, "Chaos control and solutions of fractional-order Malkus waterwheel model," Chaos, Solitons and Fractals, vol. 135, Article ID 109746, 2020.

[3] H. Wang, G. Wei, S. Wen, and T. Huang, "Impulsive disturbance on stability analysis of delayed quaternion-valued neural networks," Applied Mathematics and Computation, vol. 390, Article ID 125680, 2021.

[4] Q. X. Zhu, "pth Moment exponential stability of impulsive stochastic functional differential equations with Markovian 
switching," Journal of the Franklin Institute-Engineering and Applied Mathematics, vol. 3, no. 51, pp. 3965-3986, 2014.

[5] Z. He, C. Li, Z. Cao, and H. Li, "Stability of nonlinear variabletime impulsive differential systems with delayed impulses," Nonlinear Analysis Hybrid Systems, vol. 39, Article ID 100970, 2021.

[6] E. Hernández and D. O’Regan, "On a new class of abstract impulsive differential equations," Proceedings of the American Mathematical Society, vol. 141, no. 5, pp. 1641-1649, 2013.

[7] T. Yang, L.-B. Yang, and C.-M. Yang, "Impulsive control of Lorenz system," Physica D, vol. 110, no. 1-2, pp. 18-24, 1997.

[8] V. Lakshmikantham, D. D. Bainov, and P. S. Simeonov, Theory of Impulsive Differential Equations, World Scientific, Singapore, 1989.

[9] J. Sun and Y. Zhang, "Impulsive control of Rössler systems," Physics Letters A, vol. 306, no. 5-6, pp. 306-312, 2003.

[10] V. Lakshmikantham and J. Vasundhara Devi, "Hybrid systems with time scales and impulses," Nonlinear Analysis: Theory, Methods \& Applications, vol. 65, no. 11, pp. 21472152, 2006.

[11] A. A. Soliman, "On stability of perturbed impulsive differential systems," Applied Mathematics and Computation, vol. 133, no. 1, pp. 105-117, 2002.

[12] V. Dmitri and M. Igor, "Optimal control of a co-rotating vortex pair: averaging and impulsive control," Physica D, vol. 192, no. 1-2, pp. 63-82, 2004.

[13] C. D. Li, X. F. Liao, and X. F. Yang, "Impulsive stabilization and synchronization of a class of chaotic delay systems," Chaos, vol. 15, Article ID 043103, 2005.

[14] S. Wu, D. Han, and X. Meng, "p-moment stability of stochastic differential equations with jumps," Applied Mathematics and Computation, vol. 152, no. 2, pp. 505-519, 2004.

[15] W. P. Cao and Q. X. Zhu, "Razumikhin-type theorem for $p$ th exponential stability of impulsive stochastic functional differential equations based on vector Lyapunov function," Nonlinear Analysis-Hybrid Systems, vol. 39, Article ID 100983, 2021.

[16] Q. Zhu, "Razumikhin-type theorem for stochastic functional differential equations with Lévy noise and Markov switching," International Journal of Control, vol. 90, no. 8, pp. 1703-1712, 2017.

[17] C. Li, L. Chen, and K. Aihara, "Impulsive control of stochastic systems with applications in chaos control, chaos synchronization, and neural networks," Chaos, vol. 18, Article ID 023132, 2008.

[18] Y. J. Niu and G. Ma, "Impulsive control of stochastic system under the sense of stochastic asymptotical stability," Chinese Physics B, vol. 11, Article ID 110511, 2010.

[19] Y. Niu, D. Liao, and P. Wang, "Stochastic asymptotical stability for stochastic impulsive differential equations and its application to chaos synchronization," Communications in Nonlinear Science and Numerical Simulation, vol. 17, no. 2, pp. 505-512, 2012.

[20] Z. Chen, W. Lin, and J. Zhou, "Complete and generalized synchronization in a class of noise perturbed chaotic systems," Chaos, vol. 17, Article ID 023106, 2007.

[21] R. Sakthivel and J. Luo, "Asymptotical stability of nonlinear impulsive stochastic differential equations," Statistics \& Probability Letters, vol. 79, no. 9, pp. 1219-1223, 2009.

[22] J. Yang, S. Zhong, and W. Luo, "Mean square stability analysis of impulsive stochastic differential equations with delays," Journal of Computational and Applied Mathematics, vol. 216, no. 2, pp. 474-483, 2008.
[23] Z. Yang, D. Xu, and L. Xiang, "Exponential p-stability of impulsive stochastic differential equations with delays," Physics Letters A, vol. 359, no. 2, pp. 129-137, 2006.

[24] J. Yan and J. Shen, "Impulsive stabilization of functional differential equations by Lyapunov-Razumikhin functions," Nonlinear Analysis: Theory, Methods \& Applications, vol. 37, no. 2, pp. 245-255, 1999.

[25] Q. Wang and X. Liu, "Impulsive stabilization of delay differential systems via the Lyapunov-Razumikhin method," Applied Mathematics Letters, vol. 20, no. 8, pp. 839-845, 2007. 